\title{
Comparative study of hot embossed micro structures fabricated by laboratory and commercial environments
}

\author{
L. Lin, Y.T. Cheng, C.J. Chiu
}

\begin{abstract}
Hot embossed microstructures fabricated by laboratory and commercial environments have been studied. The laboratory process uses silicon with micropyramid patterns as the mold insert. Fine replication by using Polymethyl Methacrylate (PMMA) has been achieved. The commercial process uses electroplated nickel as the mold insert. Polyvinyl Chloride (PVC), which has a lower glass transition point than PMMA, has been used as the raw material. Fabrication results show that the laboratory process, although taking about 2 hours long per run, can successfully replicate microstructures. The commercial process which takes only 1 minute per run may be fine tuned for good replication. Atomic Force Microscope (AFM) has been used to analyze the fabricated microstructures. It is found that the root mean square (r.m.s.) roughness of PMMA is about $4 \mathrm{~nm}$ and that of PVC is about $10-15 \mathrm{~nm}$. Defects including voids and bumps on both PMMA and PVC films have been found.
\end{abstract}

1

\section{Introduction}

Hot embossing is the key and last step in the LIGA process [Bacher et al. (1995) ]. High aspect ratio microstructures can be duplicated and massively produced by using the hot embossing

Received: 25 August 1997/Accepted: 22 September 1997

L. Lin

Department of Mechanical Engineering and Applied Mechanics University of Michigan

2350 Hayward Street, Ann Arbor, MI 48109, USA

Y.-T. Cheng

Department of Electrical Engineering and Computer Science

University of Michigan

1301 Beal Avenue, Ann Arbor, MI 48109, USA

C.-J. Chiu

Institute of Applied Mechanics, National Taiwan University, Taipei, Taiwan, 106, ROC

Correspondence to: L. Lin

The laboratory hot embossing process has been conducted at Forschungszentrum Karlsruhe, Germany. The authors would like to thank Drs. W. Bacher and M. Heckele for valuable discussions and Mr. Biedermann for assisting in the laboratory hot embossing process. We would also like to thank Mr. Eric Wang at the Electron Microbeam Analysis Laboratory, the University of Michigan, Ann Arbor for helping the AFM measurements. technology with proper mold inserts. There are at least two different sources which can provide LIGA services: the LEMA project led by Bley (1995), at Karlsruhe, Germany and the LIGAMUMPs project led by Markus (1996) at MCNC, USA. Both of these services did not provide the commercial type molding process for massive production.

Hot embossing is an old technology which has to be modified in order to fabricate high aspect ratio microstructures. Several groups have been very successful in making microstructures by the micro hot embossing process. These include groups at the Forschungszentrum, Karlsruhe, Germany [Bacher et al. (1995)]; Institute of Microtechnology Mainz GmbH (IMM), Germany [Ehrfeld and Lehr (1994), Weber et al. (1996) ]; Industrial Microelectronics Center AB (IMC), Sweden [Larsson (1996), Elderstig (1996)].

Previously, a laboratory hot embossing process which used silicon mold insert made by UV lithography and anisotropic silicon wet etching has been successfully developed [Lin et al. (1996) ]. Near perfect replication of microstructures has been demonstrated. This paper explores the possibility of using a conventional hot embossing process to replace the laboratory process. Hot embossed microstructures by the commercial process are then compared with those fabricated by the laboratory process.

In this paper, both laboratory and commercial processes have used the same patterns of micro pyramids with base width of $30 \mu \mathrm{m}$. These micro pyramid structures have been successfully used as the brightness enhancement films for LCD applications [Lin et al. (1997)]. For this kind of optical applications, it is important to examine the surface quality of these hot embossed microstructures. The AFM analysis has been conduced to determine the surface roughness of these films.

\section{2}

\section{Manufacturing methods}

2.1

\section{Laboratory process}

The laboratory process has been described in detail previously [Lin et al. (1996)]. Figure 1 shows the summarized process steps. First, standard wet silicon etching process is carried out to make micro pyramids on top of silicon mold insert. The steps include: UV light lithography on silicon dioxide layer (a); etching of silicon dioxide layer to make the etching mask (b) and the anisotropic silicon etching and removal of the masking silicon dioxide layer (c). The hot embossing process is then 

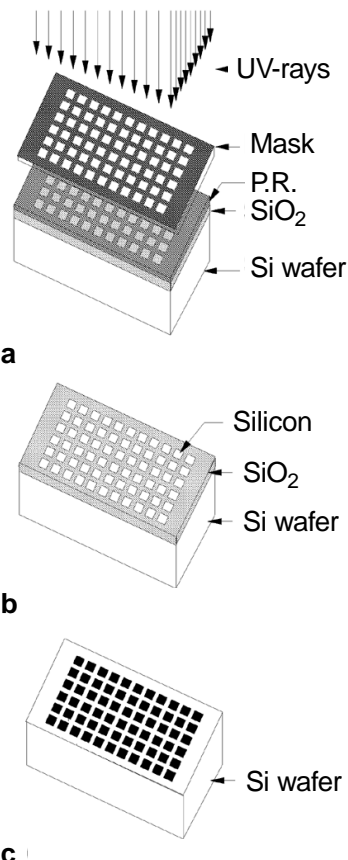
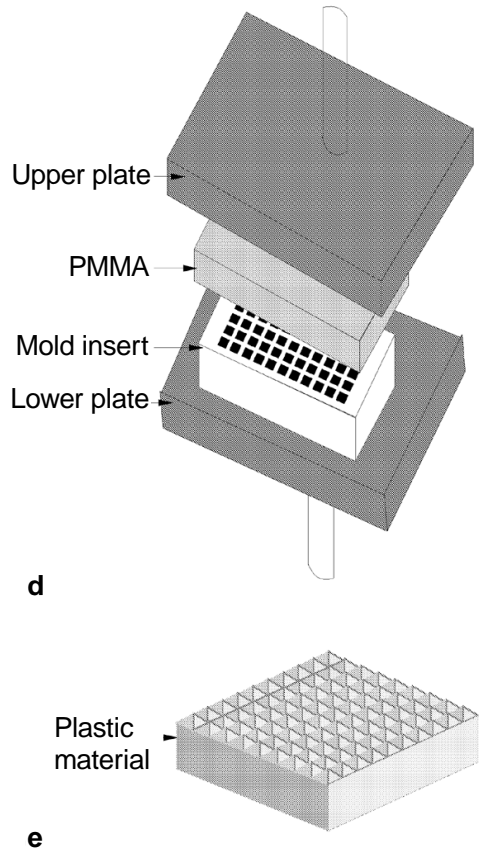

Fig. 1a-e. Fabrication sequences of the laboratory process. a lithography; b mask etching; c silicon etching; d hot embossing; e demolding

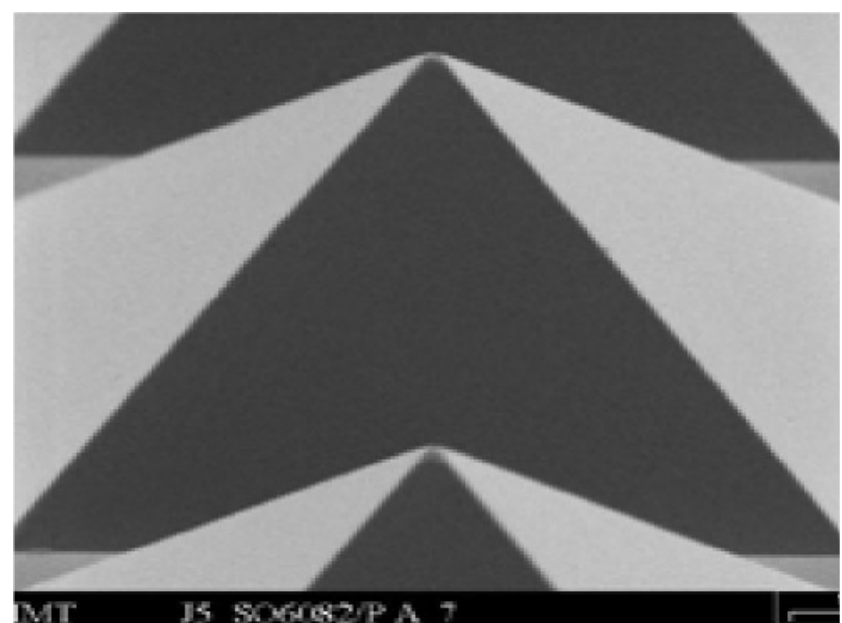

Fig. 2. Close view of a SEM micrograph showing very fine replication of PMMA micro pyramids by the laboratory process

followed as shown in the right hand side of Fig. 1. The whole four-inch silicon wafer is used as the mold inserts to transfer fine micro pyramid shapes onto the plastic material during the hot embossing process (d). PMMA (Polymethyl Methacrylate) is chosen as the raw material in the laboratory process. Finally, the fabrication process is completed after the demolding process (e). Figure 2 shows the close view SEM microphoto of the fabricated PMMA microstructures. Due to the anisotropic silicon wet etching step, the pyramid has a base width of $30 \mu \mathrm{m}$ and height of about $20 \mu \mathrm{m}$. A near perfect replication is observed.

\section{2}

\section{Commercial process}

The commercial process was contracted to a small company which has custom-made hot embossing machines. The silicon mold insert masters were first deposited with a thin layer of Ag as the seed layer for electroplating. Nickel is then electroplated on top of the silicon master until its thickness reaches about $300 \mu \mathrm{m}$. The electroplating has actually taken a two-step process in order to duplicate the same shape as the original silicon mold insert. Ag is removed from the nickel plate after the completion of the electroplating process. Figure 3 shows a SEM micrograph of the finished nickel plate. A fine pattern transfer is observed.

The nickel plate is then used in a custom-made hot embossing machine. It uses superheated vapor as the heating and water as the cooling sources. PVC films with thickness of $500-800 \mu \mathrm{m}$ are used as the raw material. The temperature is first raised to about $110^{\circ} \mathrm{C}$ and a force of about $150 \mathrm{Kg}$ is applied for $10 \sim 15$ seconds. While the applied force is maintained at the same level, the temperature is reduced in 30 seconds and the force is removed for demolding. The overall process takes only about 1 minute. Figure 4 is a photo showing

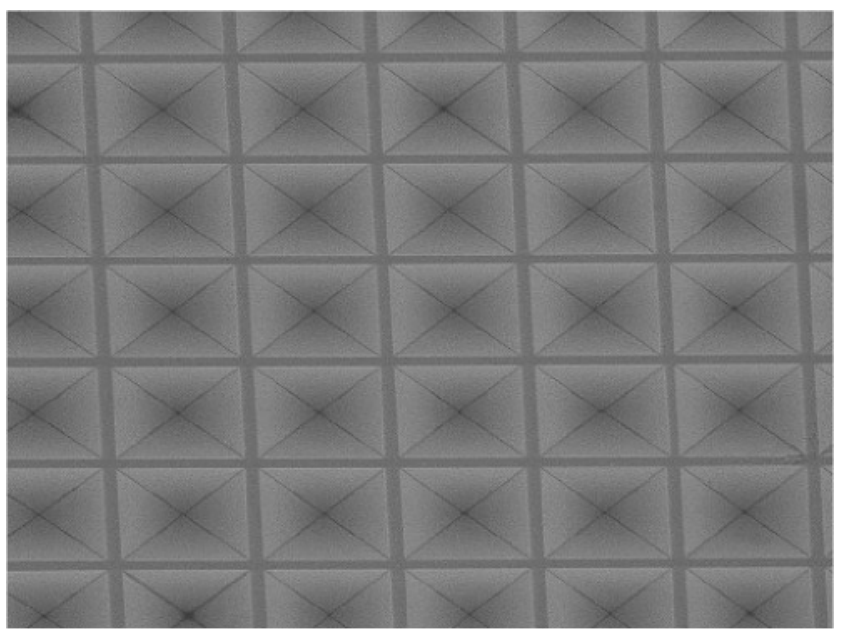

Fig. 3. A SEM micrograph showing electroplated nickel mold insert with fine pattern transfer

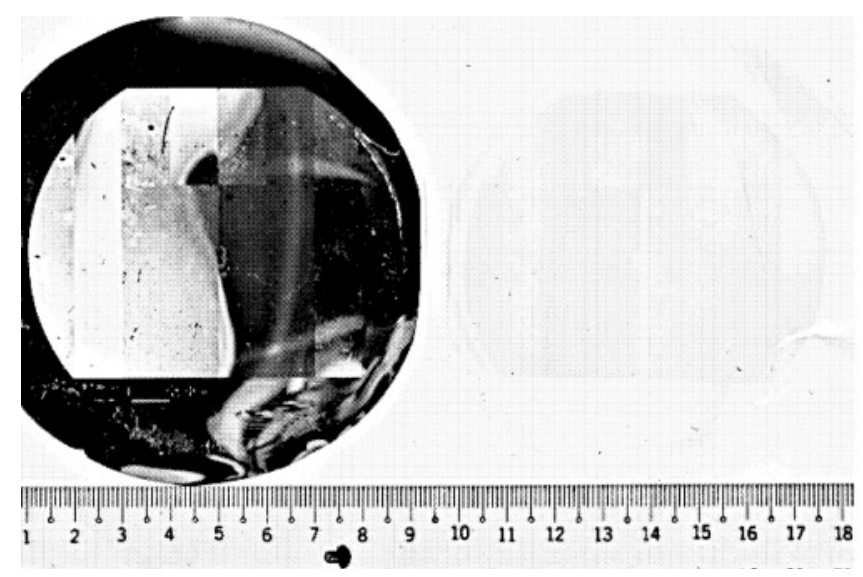

Fig. 4. A four-inch size electroplated nickel mold insert (left) and the hot embossed PVC film fabricated by the commercial process (right) 


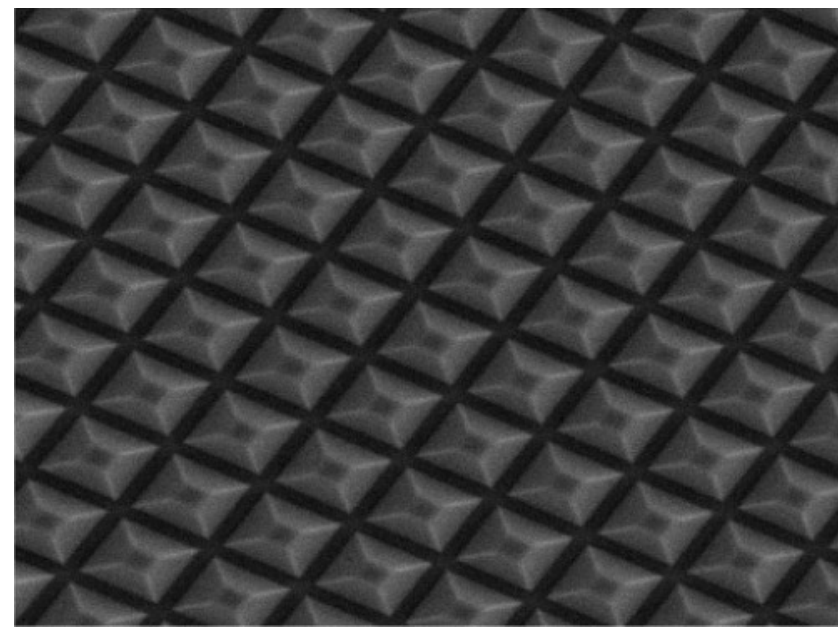

Fig. 5. A SEM micrograph of micro pyramids fabricated by the commercial hot embossing process

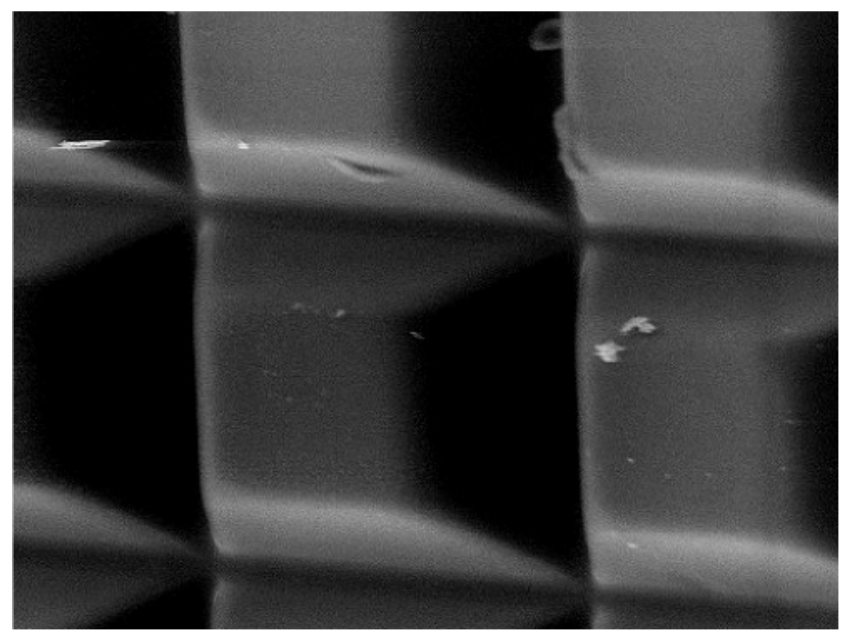

Fig. 6. A SEM micrograph showing rounded edges of microstructures made by the commercial process

the whole four-inch electroplated nickel mold insert (left) and the hot embossed pyramid microstructure (right). The SEM microphoto of fabricated PVC micro pyramids by using the commercial process is shown in Fig. 5 and Fig. 6. It is observed in both figures that the hot embossed microstructures fail to replicate the designed shapes on the mold insert.

\section{3}

\section{Results and discussions}

\section{1}

\section{Microstructure Geometry}

The geometry of the hot embossed microstructures can be qualitatively compared by examining the SEM micrographs of Fig. 2 (laboratory process) and Fig. 5 and 6 (commercial process). The laboratory process seems to be capable of making near perfect transfer of micro pyramids as shown in Fig. 2. The surfaces of these micro pyramids look smooth and no defects can be identified. It appears that the commercial process, without fine tuning, fails to produce sharp tips as shown in Fig. 5. It is estimated that the height of these pyramids is only about $15 \mu \mathrm{m}$.

Another investigation has used square patterns with base length of $100 \mu \mathrm{m}$ and height of $28 \mu \mathrm{m}$ on the silicon mold insert. Figure 6 shows the SEM micrograph of fabricated PVC microstructures by the commercial process. It is found that the height of the microstructures is about $28 \mu \mathrm{m}$ which matches well with the original depth on the mold insert. However, the edges of these microstructures are not as sharp as those fabricated by the laboratory process.

\section{2}

\section{Surface roughness}

Surface roughness is investigated by using AFM. All of the fabricated microstructures including silicon mold inserts, PMMA films and PVC films have two crystallographic planes, $\{100\}$ and $\{111\}$. The measured results are summarized in Table I. First, the r.m.s. roughness of the (100) surface on silicon is measured as a reference by using a brand new silicon wafer. The measured number of $0.06 \mathrm{~nm}$ is close to the data reported by Bhushan and Koinkar (1996). The r.m.s. roughness of silicon (111) surface is $2.2 \mathrm{~nm}$ which is much rougher than that of the (100) surface. This may be attributed to the anisotropic etching step during the fabrication of silicon mold inserts.

The r.m.s. roughness of the (100) and (111) surfaces of PMMA films are about $3.9 \mathrm{~nm}$ and $4.2 \mathrm{~nm}$ respectively. They are close to the (111) surface of the silicon mold insert. The PVC films have r.m.s. roughness of $10-15 \mathrm{~nm}$ on (100) and (111) surfaces which are larger than those of PMMA films. The slight increases of surface roughness on both PMMA and PVC films may be attributed to their intrinsic material properties or the defects observed on these films.

Two kinds of defects have been observed on the surfaces of PMMA and PVC films. Figure 7(a) shows the AFM scan of the PMMA (111) surface where voids with size of about $0.2 \mu \mathrm{m}$ in width, $0.05 \mu \mathrm{m}$ in height can be observed. Figure $7 \mathrm{~b}$ is the AFM scan of PVC (100) surface where bumps with height of about $0.3 \mu \mathrm{m}$ can be identified. Since these defects do not exist on the surface of the silicon mold insert, they are probably created during the demolding process. Since the process temperature is reduced for demolding, voids may be created if the raw material is stuck to the mold insert. Bumps may be formed for if the local temperature distribution is not uniform.

In addition to the pyramid microstructures described in this paper, hot embossing process may also be applied to fabricate

Table 1. Surface roughness measurements

\begin{tabular}{lccl}
\hline Sample ID & $\begin{array}{l}\text { Mean } \\
(\mathrm{nm})\end{array}$ & $\begin{array}{l}\text { Peak - valley } \\
(\mathrm{nm})\end{array}$ & Remarks \\
\hline Si (100) & 0.06 & 2.4 & New wafer \\
Si (111) & 2.2 & 24 & Mold insert \\
PMMA (100) & 3.9 & 78 & Laboratory process \\
PMMA (111) & 4.2 & 91 & Laboratory process \\
PVC (100) & 15 & 380 & Commercial process \\
PVC (111) & 10 & 140 & Commercial process \\
\hline
\end{tabular}



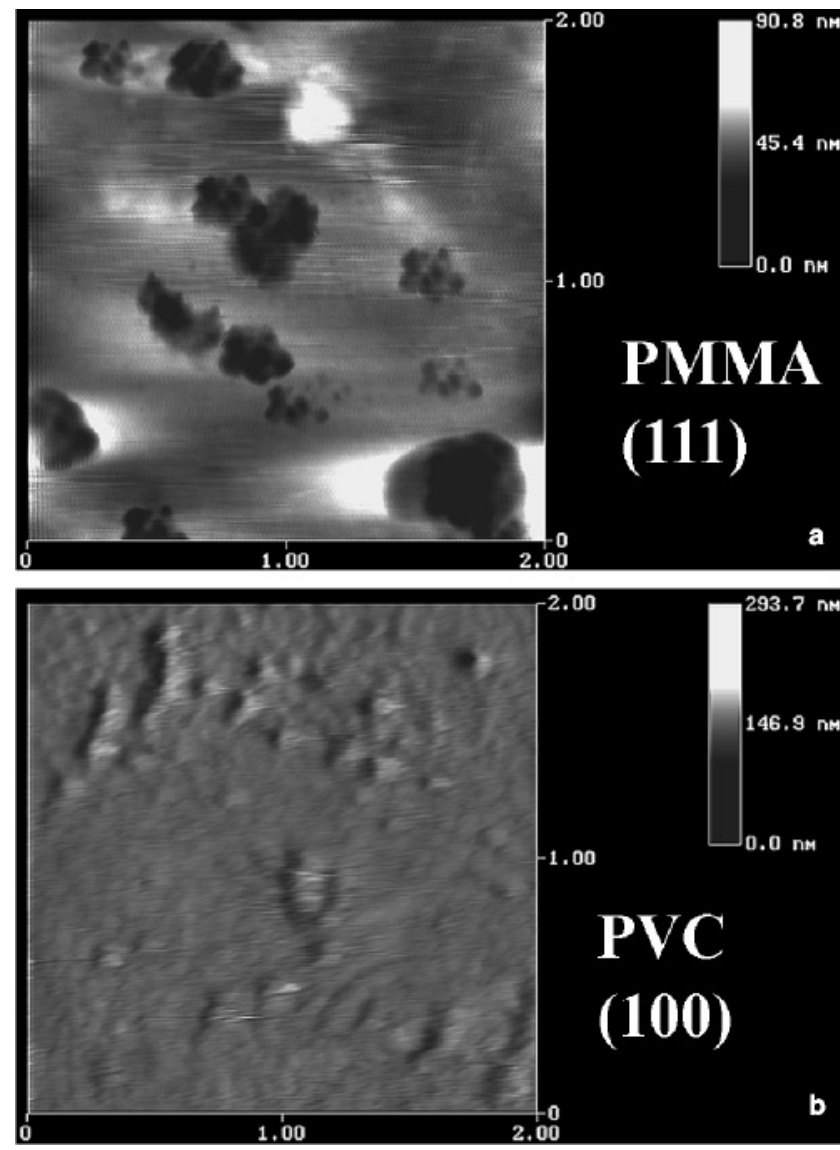

Fig. 7a, b. AFM measurements showing defects on the surfaces a PMMA (111) surface with voids; b PVC (100) surface with bumps

other micro mechanical and fluidic parts such as gears, springs, and channels. The performance of those parts depends not only on the surface roughness but also on the microtribology behavior including micro friction, microscratching and wear. Further investigations are required to characterize those properties of microstructures fabricated in both laboratory and commercial environments.

\section{3}

\section{Process improvement}

Three kinds of forces may be acting during the hot embossing process. The applied force helps the plastic material flowing into mold inserts. The other two forces, interface frictional force between the plastic material and the mold insert, and the surface tension force of the plastic material are resistant forces. Surface tension force becomes larger when the curvature of the mold insert becomes smaller. This may explain the unfinished tip shown in Fig. 5 and the rounded edges shown in Fig. 6. Increasing the process temperature (which lower the surface tension force) or increasing the applied force may alleviate this problem. These changes have been put into practice in fine tuning of both the laboratory and commercial processes. Limited commercial trial tests have been conducted to fine tune the process. It is found that when the process temperature is increased to about $120^{\circ} \mathrm{C}$ and applied force is increased to about $200 \mathrm{Kg}$, pyramids with height of about $19 \mu \mathrm{m}$ can be fabricated.

\section{4}

\section{Conclusions}

Hot embossed microstructures have been successfully fabricated under both laboratory and commercial environments. The laboratory process uses silicon as the mold insert and it takes about 2 hours for each run. The commercial process uses electroplated nickel as the mold insert and it takes only about 1 minute per run. Qualitative comparisons under SEM show that the laboratory process can well replicate microstructures defined on the silicon mold insert. The commercial process, without fine tuning, fails to fully replicate the microstructures. The problem can be improved by increasing the process temperature and the applied force. Detail AFM studies reveal that the r.m.s. surface roughness of PMMA films is about $4 \mathrm{~nm}$ and that of PVC films is about $10-15 \mathrm{~nm}$. Both of these numbers are higher than the original silicon mold insert. The reason may be attributed to the intrinsic material properties of PMMA and PVC or defects including voids and bumps observed on both films.

\section{References}

Bacher W; Menz W; Mohr J: (1995) The LIGA technique and its potential for microsystems-A survey. IEEE Trans. on Industrial Electronics, $42: 431-441$

Bhushan B; Koinkar VN: (1996) Microtribological studies of doped single-crystal silicon and polysilicon films for MEMS devices, Sensors and Actuators, A57, 91-102

Bley P: (1995) LEMA Project. Forschungszentrum, Karlsruhe, GmbH, Germany

Ehrfeld W; Lehr H: (1995) Deep X-ray lithography for the production of three-dimensional microstructures from metals, polymers and ceramics, Radiation Physics and Chemistry, 45, No. 3, 349

Elderstig H: (1996) Polymeric MST-high precision at low cost, Proceedings of MME'96, Barcelona, Spain, 1-7

Larsson O: (1996) Polymeric microstructures and replication techniques. Micro Structure Workshop 1996, 5.1-5.8

Lin Liwei; Chiu CJ; Bacher W; Heckle M: (1996) Microfabrication using silicon mold inserts and hot embossing. Proceedings of the sixth International Symposium on Micro Machine and Human Science, 67-71, October

Lin Liwei; Shia TK; Chiu CJ: (1997) Fabrication and characterization of IC processed brightness enhancement films, The 9th International Conference on Solid-State Sensors and Actuators, Transducers'97, 1421-1437 Chicago, IL, June

Markus KW: (1995), LIGAMUMPs project, MCNC, Electronics Tech. Div., 3021 Cornwallis Road, Research Triangle Park, North Carolina, USA.

Weber L; Ehrfeld W; Freimuth H; Lacher M; Lehr H; Pech B: (1996) Micro molding - a powerful tool for the large scale production of precise microstructures, SPIE Vol. 2879, 156-167 